\title{
Alkaline Phosphatase Histochemical Staining in the Study of Germinal Matrix Hemorrhage and Brain Vascular Morphology in a Very-Low-Birth-Weight Neonate
}

\author{
DIXON M. MOODY, WILlIAM R. BROWN, VENKATA R. CHALLA, AND STEVEN M. BLOCK \\ Department of Radiology [D.M.M., W.R.B.], Program in Neuroscience [D.M.M.], Department of Pathology \\ [V.R.C.], and Department of Pediatrics [S.M.B.], Bowman Gray School of Medicine of Wake Forest University,
} Winston-Salem, North Carolina 27157

\begin{abstract}
We evaluated the utility of alkaline phosphatase (AP) histochemical staining for studying intraparenchymal vascular morphology in the brain of a 31-wkgestation $(1480 \mathrm{~g})$ neonate who died of respiratory insufficiency after $23 \mathrm{~h}$. In this baby, afferent cerebral vessels (arteries, arterioles, and capillaries) stained with AP, whereas efferent vessels (venules, veins) did not. The large periventricular channels in the germinal matrix were determined to be veins, according to AP staining criteria. Arterioles connected with these large periventricular veins after passing through 4- to 6- $\mu \mathrm{m}$ capillaries. Branchings and connections of the cerebral circulation were conventional; i.e. no arterial rete or arteriovenous shunts were found. With this method of differential vascular staining, bleeding in the germinal matrix was found to be perivenous only. No dilated capillaries or arterioles were seen. Smooth muscle was identified in extrastriatal medullary arteries. This preliminary investigation suggests that AP histochemical staining is an excellent method for studying brain vascular morphology and pathology of the very-low-birthweight neonate. (Pediatr Res 35: 424-430 1994)
\end{abstract}

\section{Abbreviations}

AP, alkaline phosphatase

DWM, deep white matter

GM, germinal matrix

GMH, germinal matrix hemorrhage

VLBW, very low birth weight

Vascular lesions of the CNS are more common than any other type of neuropathology in the perinatal period.... If we are to have any chance of reducing the incidence of these conditions it is essential that we should understand their pathogenesis. This requires knowledge of the structure of the vascular bed where a particular lesion develops, at the gestational age at which it occurs, and recognition of how the structure differs from that seen at other periods of gestation. We have to know how cerebral vascular anatomy develops over the whole period in which modern perinatal care operates, that is, from 24 weeks to term. Unfortunately, there is least information on the detailed vascular anatomy of the brain for this period ... during which the whole development of the cerebral hemispheres takes place and the brain increases some hundred times in weight... Failure to recognize the need for such basic knowledge, coupled with the desire to explain all superficially

Received July 29, 1993; accepted November 10, 1993.

Correspondence and reprint requests: Dixon M. Moody, M.D., Department of Radiology, Bowman Gray School of Medicine, Medical Center Blvd., WinstonSalem, NC 27157-1088.

Supported by NIH NS 20618 Jacob K. Javits Neuroscience Investigator Award. similar lesions in terms of a simple unifying mechanism, has led to ... pathological confusion....

Since this admonition by Pape and Wigglesworth in 1979 (1), investigators have studied VLBW neonate with a variety of techniques. Serious drawbacks plague methods commonly used for the study of brain vascular morphology and pathology of the VLBW neonate.

An ideal preparation for the study of brain microvascular anatomy would have the following characteristics: it would color or opacify the vessels in a consistent and uniform manner; it would allow for thick sectioning to facilitate tracing long, complex anatomical units; it would not distort or rupture the vessels; it would allow the background tissue to be examined in relation to the vessels; and it would permit authoritative distinction between arteries and veins by means other than assessing the branching angles or following the vessels back through the tissue to a major named artery or vein, e.g. middle cerebral artery or great vein of Galen.

AP histochemical staining, a research technique formerly used for the study of adult angioarchitecture, can be used to investigate vascular-based pathology of the brain and it satisfies the criteria listed above. We recently had the opportunity to confirm the presence of the AP enzyme in a VLBW neonate, and we present our findings herein.

\section{MATERIALS AND METHODS}

The patient was a 31 -wk-gestation, 1480-g male infant whose mother was an insulin-dependent diabetic. After $48 \mathrm{~h}$ of uterine contractions for which she was treated with magnesium sulfate, the mother experienced spontaneous rupture of membranes. The amniotic fluid was lightly meconium stained. Fetal heart monitoring revealed severe bradycardia, and an emergency cesarean section was performed. The newborn's Apgar scores were 1, 3, and 7 at 1,5 , and $10 \mathrm{~min}$, respectively. His trachea was immediately intubated and he was mechanically ventilated. An initial blood gas analysis showed a pH of 7.05, arterial $\mathrm{PCO}_{2}$ of 8.379 $\mathrm{kPa}(63 \mathrm{~mm} \mathrm{Hg})$, arterial $\mathrm{Po}_{2}$ of $16.663 \mathrm{kPa}(125 \mathrm{~mm} \mathrm{Hg})$, and bicarbonate of $18 \mathrm{mEq} / \mathrm{L}$. After initial resuscitation, the patient became acutely hypoxic and pneumothorax was diagnosed. Thoracostomy tubes were placed, and aggressive medical management was continued. The patient developed pulmonary interstitial emphysema, which worsened during the next several hours. He became progressively and profoundly hypoxemic, hypercapnic, acidotic, and hypotensive; he died at the age of 23 $\mathrm{h}$. The brain was removed after a postmortem interval of $2 \mathrm{~h}$. It was placed in a refrigerator for $4 \mathrm{~h}$ to reach a consistency suitable for sectioning. No gross abnormalities were observed. The specimen submitted to our laboratory for analysis was a coronal section of the entire left cerebral hemisphere at the foramen of 

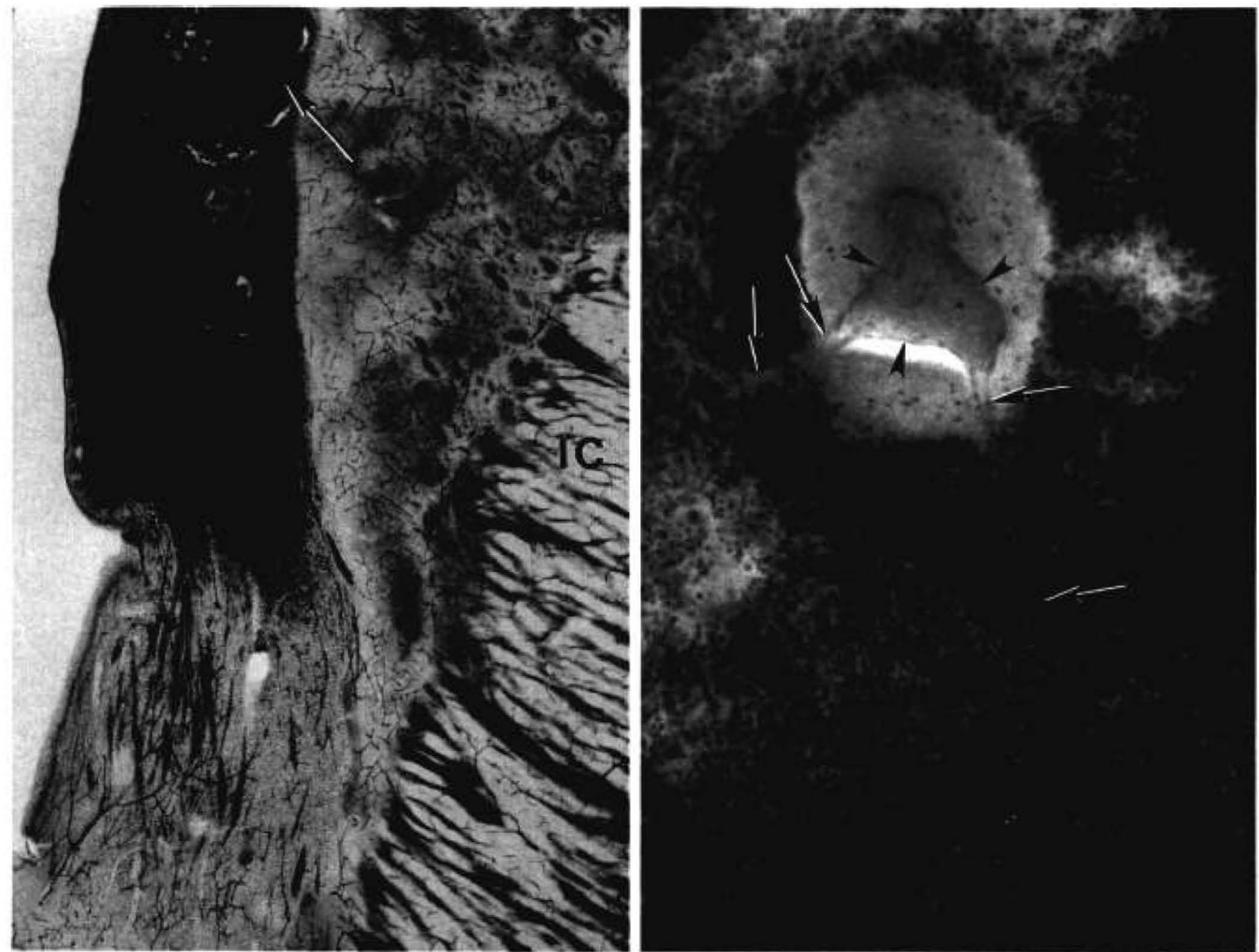

Fig. 1, left, Vascular supply to GM. A 100- $\mu$ m-thick celloidin AP-stained coronal section at the foramen of Monro from a 31-wk-gestation VLBW premature baby. The GM (blue) is thickest over the head of the caudate nucleus (top right). The lateral ventricle is on the left margin of the illustration. The afferent microvessels have AP in their endothelial cells and stain brown. The inferior portion of the GM is fed by arterioles from the recurrent artery of Heubner, and in this area most of the arterioles are traveling upward to reach their capillary bed. More superiorly, fewer arterioles are found and the veins are more plentiful. Several GMH are seen in the perivascular spaces around the veins (arrow on largest). Furthermore, GMH is more common in the mid-to-upper GM (top), where, as in this case, veins predominate, than in the inferior GM, where arterioles predominate. Unlike adults, this neonate has exuberant tissue AP in the corpus striatum and internal capsule (IC), seen as the brown, nonvascular streaks on the right half of the illustration. The smallest vessels seen are capillaries. Cresyl violet acetate and light green counterstain. Original magnification $\times 5$. Fig. 2, right, GM perivenous hemorrhage. High-power AP celloidin 100- $\mu$ m section of the same VLBW neonate, with cresyl violet acetate and light green counterstain. A GMH in the perivascular space surrounds a periventricular vein (arrowheads). Two small unstained collecting venules can be seen entering this vein (arrows). The vein can be discriminated from the afferent vessels by the lack of AP staining. This $180-\mu \mathrm{m}$ vein appears to be compressed by the perivascular hemorrhage, and the attachment of the collecting venules is "tethering" the wall. This appearance suggests a mechanism for further extension of GMH, i.e., tearing of the vessels crossing a perivenular hemorrhage. Original magnification $\times 50$.

Monro. The specimen included a thick plate of GM over the caudate nucleus head.

The block was $2 \mathrm{~cm}$ thick, and it was fixed by immersion in a cold $\left(4^{\circ} \mathrm{C}\right)$ aqueous solution containing $0.4 \%$ formaldehyde, 90 $\mathrm{mM}$ calcium chloride, and $24 \mathrm{mM}$ sodium barbital ( $\mathrm{pH} 7.0-7.2$ ) for $24 \mathrm{~h}$. (The $10 \%$ formaldehyde solution routinely used for fixation in pathology will denature the AP enzyme and render it inactive.) This block was then dehydrated in ascending grades of ethanol and was embedded in celloidin. It was serially sectioned at $100 \mu \mathrm{m}, 500 \mu \mathrm{m}$, and $1000 \mu \mathrm{m}$ on a base sledge microtome. The sections were stained for native endothelial AP by Bell and Scarrow's modification (2) of the Gomori method (3). The technique consists of incubating the sections in a medium containing calcium chloride and glycerophosphate. The endothelial AP, which is still active after this fixation and dehydration, liberates phosphate radicals, which combine with the calcium ions to form calcium phosphate. Further incubations in solutions containing lead nitrate and then ammonium sulfide convert the invisible calcium phosphate into lead sulfide, which is brown to black and suitable for light microscopic observation after optional counterstaining, then mounting and coverslipping. Most of the usual neuropathologic stains can be used as the counterstain. The lead sulfide precipitate in the endothelium attenuates soft $\mathrm{x}$-rays. High-resolution contact radiographs of the 500- and $1000-\mu$ m-thick sections were made with an ISBR-60 microradiographic unit (Softex, Tokyo, Japan) using Kodak SO-343 film (Eastman-Kodak, Rochester, NY). The radiographs were mounted on glass slides with Eukitt mounting medium and coverslips and were then examined with a light microscope.

Tissue blocks for routine paraffin sections and hematoxylin and eosin stain were also obtained from the adjacent slice of brain. These sections were used to confirm the vessel wall changes 

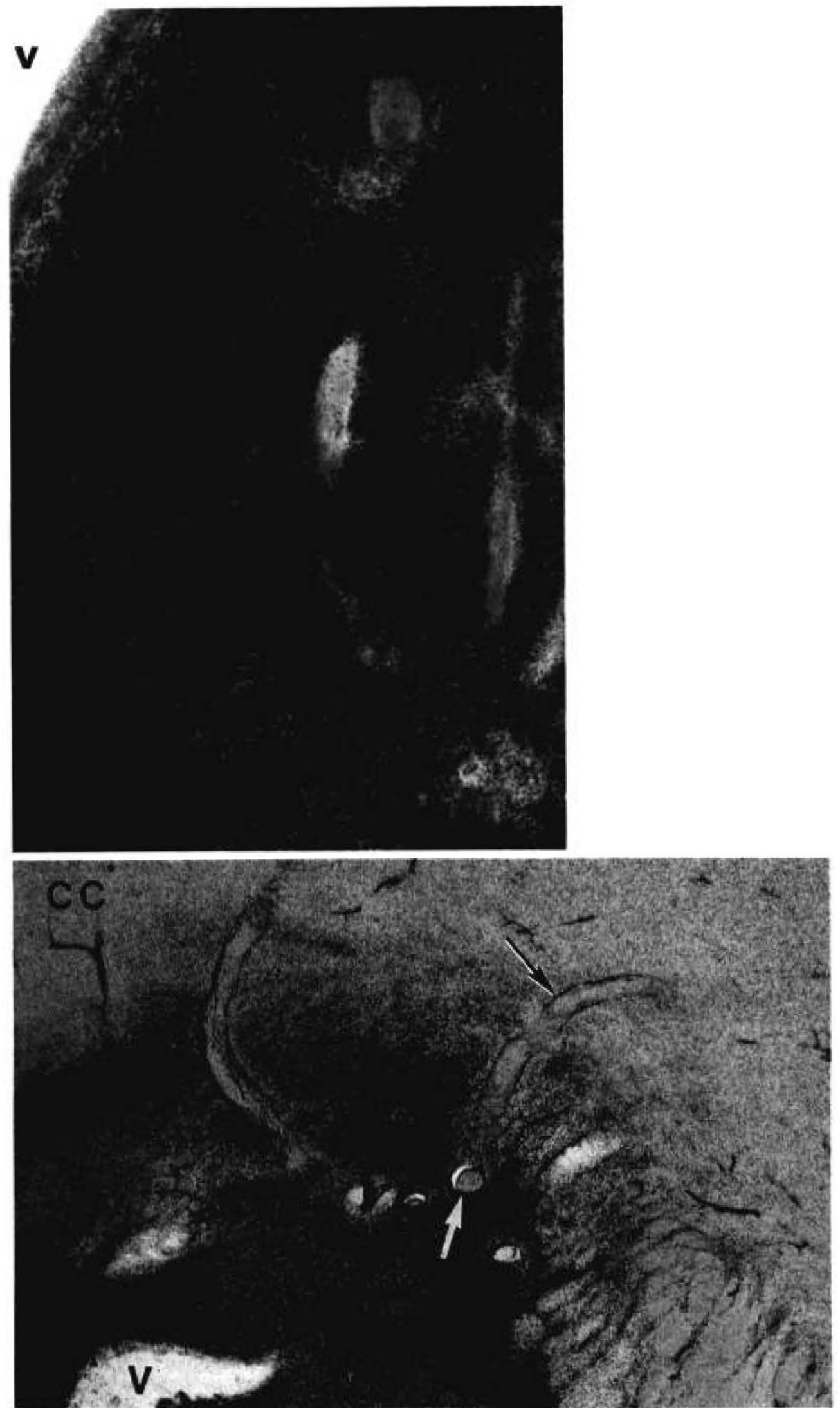

Fig. 3, top, Multifocal venous GMH. A 100- $\mu$ m-thick celloidin AP section of the GM, with cresyl violet acetate and light green counterstain. Several venous hemorrhages are seen as yellow-brown smudges, widening the perivascular spaces (center and to right). The AP-stained arterioles and capillaries are not associated with hemorrhage. The lateral ventricle $(V)$ is in the upper left. Original magnification $\times 25$. Fig. 4, bottom, Periventricular veins connect with medullary veins. An AP-stained celloidin 100- $\mu \mathrm{m}$ section of the same VLBW neonate, with cresyl violet acetate and light green counterstain. $V$, ventricle; $C C$, corpus callosum. Germinal matrix is dark cellular material to bottom and left. There are connections between periventricular veins (white arrow on one example) and the transcerebral medullary veins (highlighted arrow on one example). Such vessels may also drain medially toward the Galenic drainage system. The dark material adjacent to these veins consists of germinal cells (small black arrows) migrating outward, not vascular endothelial AP reaction product. Note also that the only afferent (stained) vessels approaching the ventricles at this level are the smallest arterioles and capillaries. Original magnification $\times 10$. 


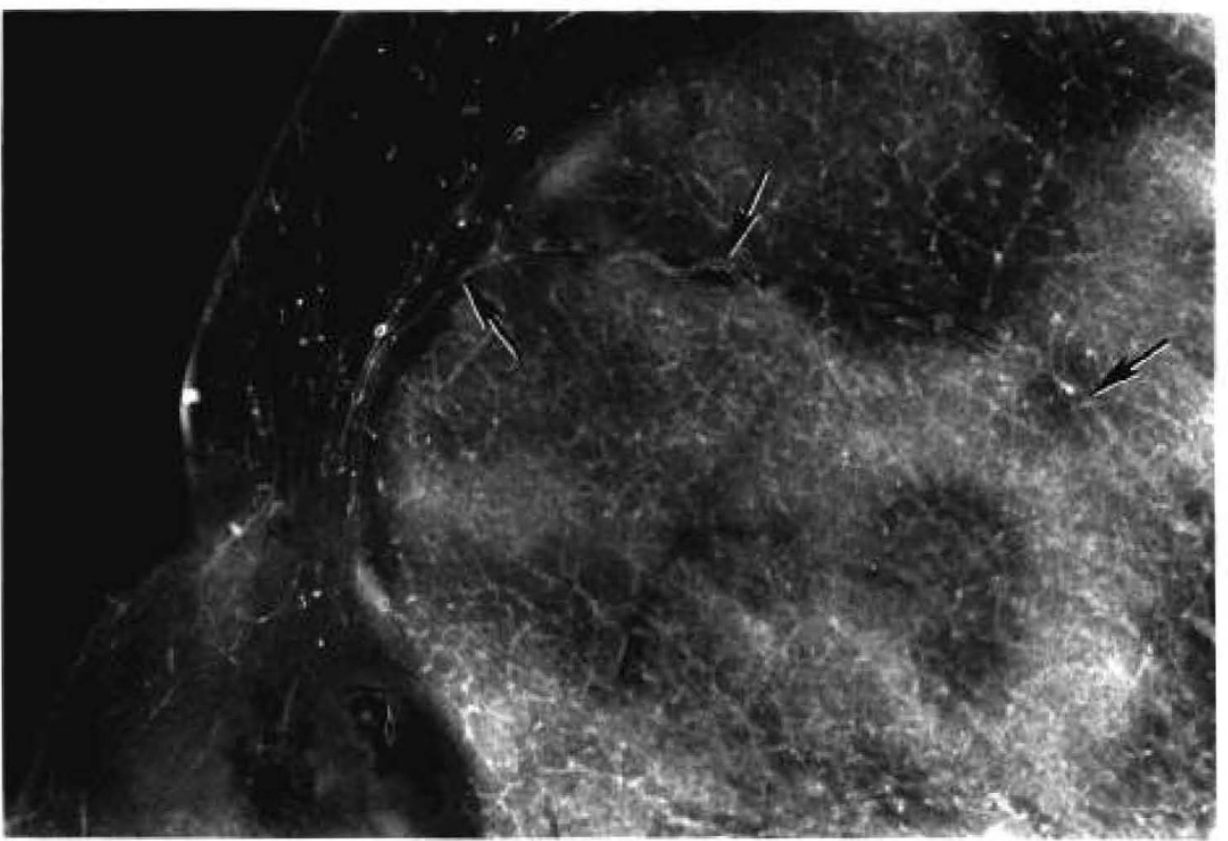

Fig. 5. Microradiograph of a 500 - $\mu$ m-thick, AP-stained coronal section. Distal branches of the lateral striate artery (arrows) ramify into the GM over the head of the caudate nucleus. The presence of modest tissue AP in the corpus striatum, but not in the GM, produces a lighter background in the former tissue. Tissue AP can be observed as brown, streaky, nonvascular densities in Figure 1 as well. The ventricle is to the left. Original magnification $\times 10$.

and overall correlation with the findings observed on the histochemical preparations.

\section{RESULTS}

Linear shrinkage of the tissue was $15-20 \%$. The celloidin embedding material allowed uniformly thick sections to be cut without cracking or distortion of the tissue.

Most of the arterioles supplying the GM at this level (foramen of Monro) arose from the recurrent artery of Heubner. The inferior part of the GM contained predominantly arterioles, a few capillaries, and even fewer small venules and veins (Fig. 1). This inferior GM area was traversed by many arterioles passing superiorly to reach their capillary bed in the GM adjacent to the inferior portion of the caudate nucleus (Fig. 1). In the mid-GM the numbers of arterioles, capillaries, and thin-walled periventricular veins were approximately equal (Figs. 1-3). More superiorly, thin-walled veins predominated over arterioles (Fig. 4). At the lateral angle of the lateral ventricle, the periventricular veins converged, connected with transcerebral medullary veins, and fanned out toward the cortical surface (Fig. 4).

In the region of the mid and upper caudate nucleus, terminal arteriolar branches of the lateral striate arteries entered the GM to supply the capillary bed (Fig. 5). A VLBW neonate of 31-wk gestation has thin GM tissue on the inferior surface of the corpus callosum. This GM derives its vascular supply from the terminal branches of the callosal penetrating arterioles. These vessels, like the artery of Heubner, arise from the anterior cerebral artery.

In this baby, the arteries, arterioles, and capillaries had excessively strong AP stain, and there was considerable reaction product in the corpus striatum because of tissue AP (Figs. 1 and 5). This tissue AP was separate from vascular AP. Although large periventricular veins in the GM exhibited faint AP staining after lengthy (excessive) incubation with the substrate, they were easily distinguished from the more densely staining arterioles and capillaries (Fig. 6). Elsewhere, the veins could be seen by the counterstaining used (Fig. 6).

Some extrastriatal medullary arteries/arterioles could be traced the entire length from the cortex to the GM in several contiguous $100-\mu \mathrm{m}$ sections. Striate arteries and arterioles, as well as extra- striatal medullary arterioles, had rudimentary smooth muscle in their walls, as identified by Gill's hematoxylin counterstain.

No arteriolar-to-arteriolar or arteriolar-to-venous shunts or vascular rete could be identified, although connections between arterioles via the continuous capillary bed were seen often (Fig. 7). The terminal vascular bed had conventional branchings and connections, i.e. arteriole-to-capillary-to-postcapillary venule-tocollecting venule-to-vein (Figs. 2 and 7).

There was one modest-sized GMH measuring $5 \mathrm{~mm}$ in diameter, but this hemorrhage did not extend into the DWM or ventricle. No hydrocephalus or choroid plexus hemorrhage was identified. There were six smaller GMH confined to the perivascular spaces surrounding veins (Figs. 1-3). All of the arterioles and capillaries that were traced appeared normal. These vessels were demonstrated both in the ependymal aspect and the lateral aspect of the main GMH. Intact capillaries were traced to within $10 \mu \mathrm{m}$ of the ependymal lining (Fig. 3).

\section{DISCUSSION}

The AP staining technique, first described by Gomori (3) and later modified by Bell $(2,4)$, precipitates lead sulfide salt at the site of the ectoenzyme, nonspecific AP, in the luminal and abluminal plasma membranes of endothelial cells of small arteries, arterioles, and capillaries, but not veins. The veins are visualized by means of counterstains (e.g. cresyl violet acetate/ light green, Gill's hematoxylin). Because of the black lead sulfide precipitate, extensive segments of the vascular tree can be analyzed with the light microscope in thick $(100-\mu \mathrm{m})$ celloidin sections. Thicker sections (500-1000 $\mu \mathrm{m}$ ), displaying even more complete vascular patterns, can be microradiographed because the lead salt also attenuates soft $x$-rays (Fig. 5). The resulting radiographs can be viewed with magnification as high as $100 \times$ before image degradation occurs. In our study, the lack of sharpness was caused by the focal spot configuration rather than film emulsion graininess. Capillaries could be seen clearly with the $\mathrm{x}$-rays (Fig. 5).

We believe for several reasons that the AP method of staining the vessel walls of the brain microvasculature is superior to methods that require staining of intravascular contents (i.e. blood) or postmortem injection of barium, india ink, colored 

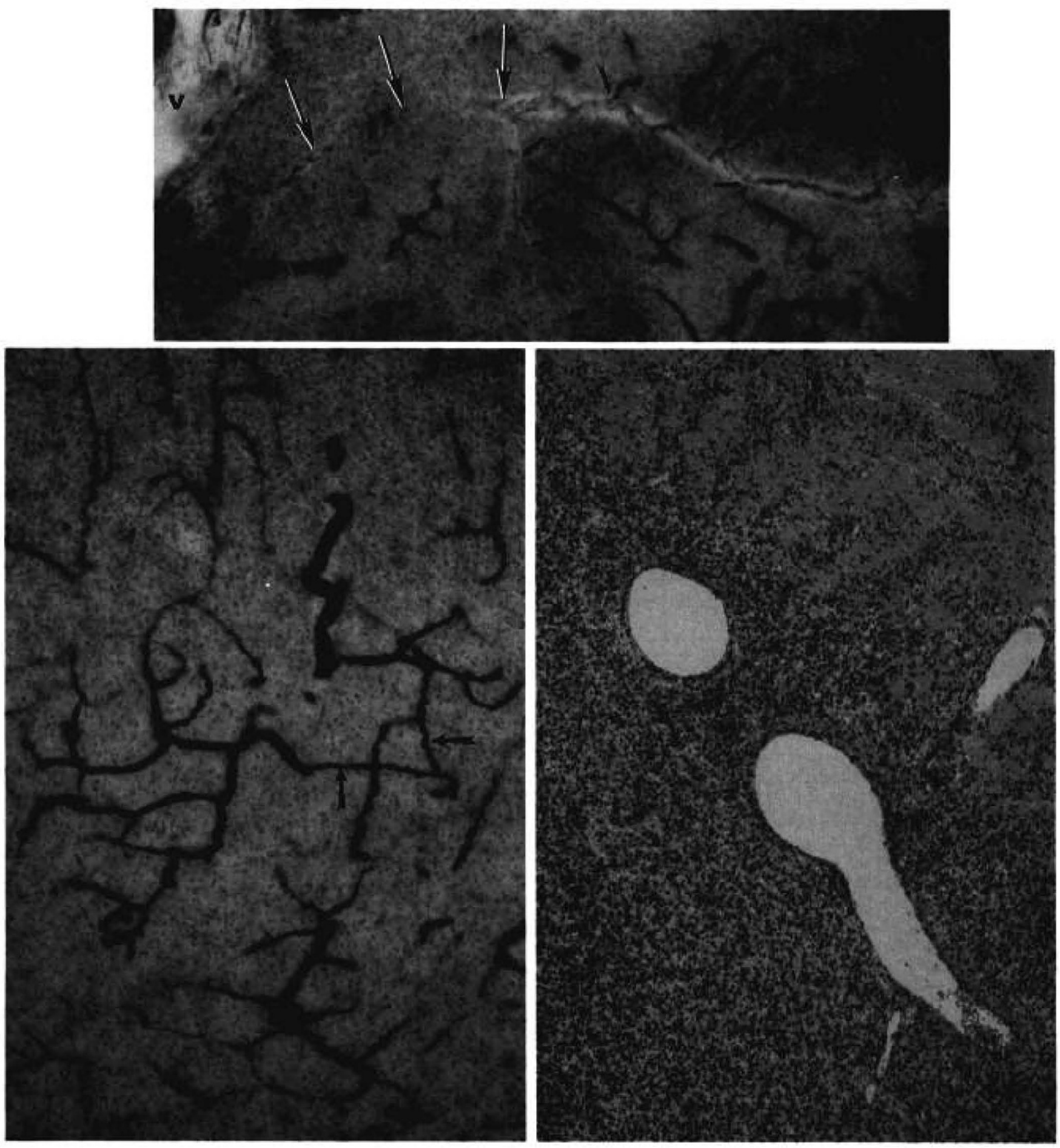

Fig. 6, top, Capillaries, postcapillary venules, and a collecting venule from the VLBW neonate in Figure 1. An AP-stained 100- $\mu$ m-thick celloidin section with cresyl violet acetate and light green counterstain. The AP-stained capillaries can be followed into postcapillary venules without AP staining (small arrows). The venules, in turn, drain into a collecting venule. This collecting venule crosses from right to left (large highlighted arrows) into the GM, where it connects with a larger thin-walled GM periventricular vein ( $V$ ). Despite faint AP staining, the larger GM vein can easily be discriminated from the arterioles and capillaries. In this instance, as well as in others observed, the vascular bed is classic, i.e. arteriole-tocapillary-to-vein; furthermore, the arterioles do not connect directly into the large (fragile?) thin-walled veins. Original magnification $\times 25$. Fig. 7 , lower left, Continuous capillary bed. A $100-\mu \mathrm{m}$-thick AP-stained coronal section with cresyl violet acetate and light green counterstain. No unconventional connections were found between arterioles and veins or arterioles-to-arterioles directly. The continuous capillary bed, wherein arterioles connect to arterioles via capillaries (arrows on one example), was common throughout. In this regard, i.e. regarding the branching and connections, this 31-wk VLBW premature infant is similar to the 77 adults, 4 monkeys, 12 dogs and 6 rats we have investigated with this technique. Original magnification $\times 25$. Fig. 8, lower right, Large $(50-$ to $200-\mu \mathrm{m})$ channels suspended in a sea of germinal matrix cells in a different VLBW human neonate than the one illustrated in Figures 1-7. From this routine 5- $\mu \mathrm{m}$-thick paraffin hematoxylin and eosin-stained section, it is impossible to determine whether the vessels are capillary sinusoids or veins because the wall is only one endothelial cell thick, and its connections and origins are difficult to trace. Original magnification $\times 25$. 
latex, or plastic. With the AP method, the arterial side of the microvasculature can be distinguished directly from the venous (the former stains; the latter does not). Thick specimens can be examined with light microscopy and even thicker ones with $\mathrm{x}$ rays. The background neural tissue can be examined with standard or special counterstains. There is no leaking of intravascular contents at the cut section surface to compromise the observations. Finally, vascular distortion, incomplete filling, air bubbles, aneurysms, rupture, and other artifacts of injection are avoided. This feature was of critical importance in this case because we were searching for points of rupture.

Previous investigators used AP staining to analyze the angioarchitecture in fetal mammalian (5), adult human (6), and fetal and neonatal human (7) CNS tissue, primarily cortex. We have found the AP staining method useful in the study of pathologic changes in the microvasculature of the adult human brain, including deep structures $(8-16)$. There is no report of the use of AP vascular staining to investigate the primitive $G M$ or periventricular cerebral area.

The principal objective of this communication is to document the efficacy of the AP technique in a VLBW neonate. However, after study of hundreds of deep arterial/arteriolar systems in this series of coronal sections of the left cerebral hemisphere, we wish to comment on some long-held beliefs regarding the DWM and GM vasculature. Although true for this single patient, these findings must be regarded as preliminary at this early stage of our investigation.

Germinal matrix vascular morphology. Previous investigations of the GM (17-19), using routine 5- to 15- $\mu \mathrm{m}$ paraffin-embedded sections, have limited ability to trace vessels and branchings. The classic fingerprint of the afferent and efferent vessels (smooth muscle in arterial/arteriolar walls and collagen in the adventitia of veins) is seldom present in the brain of the VLBW neonate.

Using injection techniques, a number of investigators have studied the GM $(1,20,21)$ and DWM $(22,23)$ vascular morphology of the VLBW neonate. These techniques, which overcome the limitation of paraffin embedding, are nevertheless flawed because of incomplete filling, air bubbles, rupture artifacts, and vascular distortions. Most importantly for our purposes, their inability to discriminate consistently between arteries and veins complicates interpretation. Well-injected, thick sections produce an unintelligible maze of vessels, both arteries and veins, a point amply illustrated in the material presented by Pape and Wigglesworth (1). When attempting to obtain a completely injected arterial system, it is difficult to prevent the arterially injected material from passing through the capillaries to fill veins.

Intraparenchymal vascular anastomoses. Another controversy concerns the presence of precapillary arteriolar-to-venous shunts $(1,24)$ or arteriolar-to-arteriolar anastomoses $(21,22)$, both of which we have been unable to document in adult brains, in spite of our vigorous attempts to do so over $9 \mathrm{y}$ of investigation (10). Although Pape and Wigglesworth (1) described an "immature vascular rete in the subependymal matrix" (our understanding of a rete is a vessel that breaks up into a network and then reforms into the same kind of vessel) and Nelson et al. (23) described shunts between vascular "channels" in midgestation in the cerebrum, none of these vascular connections were found in this VLBW premature baby. Such shunts are common in the skin and other organs, and communication between adjacent capillaries is present in the adult human brain (10). However, even when a capillary was connected to two arterioles, it first had to pass a junction with a postcapillary venule (for this reason, intraparenchymal collateral flow is weak and inefficient, because putative collateralizing flow prefers to select the venule rather than the adjacent arteriole). Such capillary connections were seen (Fig. 7) in the present case, but arterial-to-venous shunts were not. Arterial-to-venous shunts would be an important mechanism for transmitting arterial pressure directly into fragile periventricular GM veins, and the AP microvascular morphologic technique should provide a definite answer as to whether or not these shunts exist in the preterm infant.

Periventricular vessels: capillaries or veins? Reports published in the pathology literature differ on how to classify the large vessels composed of a single layer of endothelial cells "suspended in a sea of germinal cells" (Fig. 8) in premature infants. Although Rorke (17) and Sotrel and Lorenzo (25) call them capillaries, our AP-stained preparations (Figs. 1-4) confirm that they are, in this instance, veins. Kuban and Gilles (22) and Nelson et al. (23) could not identify vascular-wall collagen or smooth muscle in any periventricular or white matter vessels of premature or term human babies. Therefore, they described these structures as channels instead of arteries, capillaries, or veins. These periventricular veins converge at the lateral angle of the lateral ventricle. There they connect with transcerebral medullary veins, fanning out toward the brain surface (Fig. 4). We believe that, in some studies $(26,27)$, these veins have been mistaken for centrifugal arteries, and we have published evidence using the AP staining method to support our claim that these are veins (10).

Site of bleeding in GMH. Some believe that bleeding in the GM occurs at arteriolar or capillary sites $(1,28,29)$. Others suspect the veins to be the primary site of bleeding $(19,30,31)$. In this case, several GMH were found, all of which originated from periventricular veins. This determination was based on the indirect evidence that extravascular red blood cells were found in the venous perivascular spaces but not in those associated with arterioles or capillaries (Figs. 1-3). Perivenous hemorrhage compresses veins and therefore is a putative mechanism for focal circulatory stagnation. These hemorrhages also stretch and possibly tear venular tributaries at their attachments to the larger veins (Fig. 2).

In conclusion, we have established AP enzymatic activity in the endothelial cells of GM and DWM afferent vessels of the VLBW neonate reported herein. The GMH in this baby was established as venous in origin. AP staining, combined with counterstaining of the background tissue and large, thick sections, greatly increases opportunities to locate, count, trace, and analyze vascular and related parenchymal lesions. On the basis of our early studies, we have presented preliminary observations on some widely held beliefs regarding the angioarchitecture of this region. Our results suggest that our AP histochemical staining method will be useful in a comprehensive investigation of GM vascular morphology and site(s) of vascular injury in GMH.

Acknowledgments. The authors thank Julianne R. Berckman, Donna S. Garrison, Ph.D., and Nancy A. Ragland for expert help in preparation of the manuscript. The assistance of Dr. Patrick Lantz in securing the brain specimen is appreciated. We wish to acknowledge the Siemens Medical Corporation for the cost of the color illustrations.

\section{REFERENCES}

1. Pape KE, Wigglesworth JS 1979 Haemorrhage, ischaemia and the perinatal brain. Clin Dev Med 69/70:11-132

2. Bell MA, Scarrow WG 1984 Staining for microvascular alkaline phosphatase in thick celloidin sections of nervous tissue: morphometric and pathologic applications. Microvasc Res 27:189-203

3. Gomori G 1941 Distribution of phosphate in normal organs and tissues. J Cell Physiol 17:71-83

4. Saunders RL, Bell MA 1971 X-ray microscopy and histochemistry of the human cerebral blood vessels. J Neurosurg 35:128-140

5. Scharrer E 1950 A technique for the demonstration of the blood vessels in the developing central nervous system. Anat Rec 107:319-327

6. Bannister RG, Romanul FCA 1963 The localization of alkaline phosphatase activity in cerebral blood vessels. J Neurol Neurosurg Psychiatry 26:333340

7. Norman MG, O'Kusky JR 1986 The growth and development of microvasculature in human cerebral cortex. J Neuropathol Exp Neurol 45:222-232

8. Moody DM, Bell MA. Challa VR 1988 The corpus callosum, a unique whitematter tract: anatomic features that may explain sparing in Binswanger disease and resistance to flow of fluid masses. AJNR 9:1051-1059

9. Challa VR, Moody DM, Bell MA 1992 The Charcôt-Bouchard aneurysm controversy: impact of a new histologic technique. J Neuropathol Exp Neurol $51: 264-271$ 
10. Moody DM, Bell MA, Challa VR 1990 Features of the cerebral vascular pattern that predict vulnerability to perfusion or oxygenation deficiency: an anatomic study. AJNR 11:431-439

11. Bell MA, Moody DM, Angelo JN, Challa VR, Johnston TC 1987 Alkaline phosphatase staining of leaky blood vessels in human post mortem area postrema and choroid plexus. Soc Neurosci Abstr 13:1251(abstr)

12. Moody DM, Santamore WP, Bell MA 1991 Does tortuosity in cerebra arterioles impair down-autoregulation in hypertensives and elderly normotensives? A hypothesis and computer model. Clin Neurosurg 37:372-387

13. Moody DM, Bell MA, Challa VR 1992 Regional cerebral vulnerability to perfusion deficits in the elderly. NINS-New Issues in Neurosciences: Basic and Clinical Approaches 4:133-145

14. Challa VR, Bell MA, Moody DM 1990 A combined hemotoxylin-eosin, alkaline phosphatase and high-resolution microradiographic study of lacunes. Clin Neuropathol 9:196-204

15. Abernethy WB, Bell MA, Morris M, Moody DM 1993 Microvascular density of the human paraventricular nucleus decreases with aging but not hypertension. Exp Neurol 121:270-274

16. Moody DM, Bell MA, Challa VR, Johnston WE, Prough DS 1990 Brain microemboli during cardiac surgery or aortography. Ann Neurol 28:477486

17. Rorke LB 1982 Pathology of Perinatal Brain Injury. Raven Press, New York, pp 13-140

18. Friede RL 1989 Developmental Neuropathology, 2nd Ed. Springer-Verlag. Berlin

19. Volpe JJ 1989 Intraventricular hemorrhage in the premature infant: curren concepts. Part 1. Ann Neurol 25:3-11
20. Takashima S, Mito T, Ando $\mathrm{Y} 1986$ Pathogenesis of periventricular white matter hemorrhages in preterm infants. Brain Dev 8:25-30

21. Nakamura Y, Okudera T, Hashimoto T 1991 Microvasculature in germinal matrix layer: its relationship to germinal matrix hemorrhage. Mod Pathol $4: 475-480$

22. Kuban KCK, Gilles FH 1985 Human telencephalic angiogenesis. Ann Neurol 17:539-548

23. Nelson Jr MD, Gonzalez-Gomez I, Gilles FH 1991 The search for human telencephalic ventriculofugal arteries. AJNR 12:215-222

24. Hasegawa T, Ravens JR, Toole JR 1967 Precapillary arteriovenous anastomoses: "thoroughfare channels" in the brain. Arch Neurol 16:217-224

25. Sotrel A, Lorenzo AV 1989 Ultrastructure of blood vessels in the ganglionic eminence of premature rabbits with spontaneous germinal matrix hemorrhages. J Neuropathol Exp Neurol 48:462-482

26. Van den Bergh $R 1969$ Centrifugal elements in the vascular pattern of the deep intracerebral blood supply. Angiology 20:88-94

27. de Reuck J 1971 The human periventricular arterial blood supply and the anatomy of cerebral infarctions. Eur Neurol 5:321-334

28. Hambleton G, Wigglesworth JS 1976 Origin of intraventricular haemorrhage in the preterm infant. Arch Dis Child 51:651-659

29. Pasternak JF, Groothuis DR, Fischer JM, Fischer DP 1983 Regional cerebral blood flow in the beagle puppy model of neonatal intraventricular hemorrhage: studies during systemic hypertension. Neurology 33:559-566

30. Leech RW, Kohnen P 1974 Subependymal and intraventricular hemorrhages in the newborn. Am J Pathol 77:465-476

31. Towbin A 1968 Cerebral intraventricular hemorrhage and subependyma matrix infarction in the fetus and premature newborn. Am J Pathol 52:121 139 